\title{
Disorder-induced topological transitions in multichannel Majorana wires
}

\author{
B. Pekerten, ${ }^{1, *}$ A. Teker, ${ }^{1}$ O. Bozat, ${ }^{1}$ M. Wimmer,${ }^{2}$ and İ. Adagideli ${ }^{1},{ }^{\dagger}$ \\ ${ }^{1}$ Faculty of Engineering and Natural Sciences, Sabancu University, Orhanl - Tuzla, 34956, Turkey \\ ${ }^{2}$ QuTech and Kavli Institute for Nanoscience, Delft University of Technology, Lorentzweg 1, 2628 CJ Delft, The Netherlands
}

(Dated: September 2, 2015)

\begin{abstract}
In this work, we investigate the effect of disorder on the topological properties of multichannel superconductor nanowires. While the standard expectation is that the spectral gap is closed and opened at transitions changing the topological property of the ground state, we show that the closing and opening of a transport gap can also cause topological transitions, even in the presence of (localized) states at both sides of the transition. Such transport gaps, induced by disorder, can thus change the topological index, driving a topologically trivial wire into a nontrivial state. We focus on nanowires exhibiting $p$-wave superconductivity as well as Rashba semiconductor nanowires in proximity to a conventional superconductor, and obtain analytical formulas for topological transitions in these wires, valid for generic realizations of disorder, generalizing earlier results. Full tight-binding simulations show excellent agreement with our analytical results without any fitting parameters.
\end{abstract}

PACS numbers: 74.78.Na, 74.45.+c, 71.23.-k

\section{INTRODUCTION}

Topologically nontrivial phases are exotic states of matter that have an electronic band gap in their bulk and protected gapless excitations at their boundaries 13 . Superconductors, being quasiparticle insulators, also feature topological phases with a quasiparticle gap in the bulk and edge or surface excitations. For 1D systems, these edge states are fermionic zero-energy modes called Majorana states 4 . These states attracted intense attention owing to their non-Abelian nature which led to the proposals to use them as topological qubits immune to decoherence ${ }^{9 \mid 10}$. Although predicted to appear in exotic condensed matter systems with unconventional superconducting pairing 11 , recent proposals ${ }^{17}[19]$ involving hybrid structures of more conventional materials have appeared 20 . This led to the recent conductance measurements done on a proximity coupled InSb nanowire ${ }^{21}$, which showed some evidence of Majorana end states in the form of zero bias conductance peaks. Other experiments reported further observations of zero bias peaks in similar settings $22-25$. Very recently, scanning-tunneling spectroscopy experiments on magnetic adatom chains on a conventional superconductor reported ZBPs at the ends of the chain 26. While it is compelling to interpret the observation of these ZBPs as signatures of Majorana states, the issue is still under intense discussion 27 .

Semiconductor nanowire structures that are proximitycoupled to superconductors are technologically attractive platforms for Majorana physics. However, disorder has been prominently present in all such experimental samples to date. This led to a renewed interest in disordered superconducting wires, particularly focusing on the effects of disorder on Majorana states 28 47. These works focused mostly on disordered $p$-wave superconducting wires (PW wires) and showed that disorder is detrimental to the spectral gap as well as to the formation of Majorana fermions in both strictly 1D systems $s^{28|29| 35|41| 46 \mid 47}$ and in multichannel wires $34|40| 42 \mid 48$.
In a recent study on the experimentally relevant hybrid structures with Rashba spin-orbit interaction (SOI) proximity coupled with an $s$-wave superconductor (RSW nanowires for short), some of us showed that disorder need not be detrimental to and in fact can even create topological order in $1 \mathrm{D}$ wires ${ }^{46}$. We are not aware of any study of the effects of disorder on the topological properties of the multichannel RSW nanowires.

In this Manuscript, we investigate the topological properties of disordered multichannel RSW and PW superconductor nanowires. The standard expectation for these nanowires is that if their topological state is switched by modifying certain external parameters (such as gate potential or magnetic field), the spectral gap will close and open concomitantly with the transition. Here we show that for disordered nanowires, the closing and opening of a transport gap can also cause topological transitions, even in the presence of (localized) states at both sides of the transition. We thus extend our earlier work ${ }^{46}$ to multichannel wires. We first reproduce results showing reentrant behavior in multichannel $\mathrm{PW}$ wires ${ }^{42}$. Next we derive analytical expressions of the topological phase diagram for the experimentally relevant quasi-1D RSW nanowire. In particular, we find new topological regions in the phase diagram showing up as additional reentrant behavior on top of those predicted from PW wire model. The RSW wires under high magnetic fields (enough to completely spin-polarize the wire) are well described by the PW wire model. In agreement with the earlier results, however, the topological order in this limit is fragile, and disorder is always detrimental to it. In contrast, topological order in the low magnetic field limit, which is robust against disorder, requires full description of all spin bands, as shown by our analytical results (see Fig. 3). Our numerical simulations using a tight-binding (TB) approach show very good agreement with our analytical formulas.

This Manuscript is organized as follows: We begin the next section by reviewing the system in question as well 


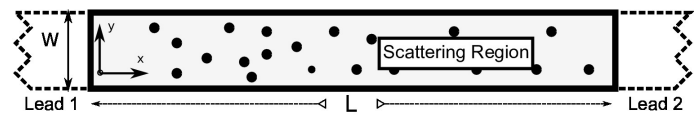

FIG. 1. The quasi-1D nanowire of width $W$, which is a PW or an RSW topological superconductor with a Gaussian disorder having an average value $\left\langle V_{\text {ave }}\right\rangle=0$. In the leads, we take $\alpha$, $\Delta$ and $V(x, y)$ to be zero, making the leads metallic. Our analytical results assume a semi-infinite wire $(L \rightarrow \infty)$, whereas in our numerical full tight-binding calculations we use wires of length $L \gg l_{\mathrm{MFP}}, \xi$.

as introducing our numerical and analytical methods. In subsection IIA, we consider a spin-orbit coupled quantum wire in proximity to RSW superconductor and its fully spin polarized limit (effectively a PW superconducting quasi-1D nanowire), extending previous work ${ }^{42 \mid 46}$ to multichannel nanowires. We show that a transport gap, as well as a spectral gap, can generate and protect Majorana states. In the next subsection (IIB), we investigate the case of RSW topological superconductor (TS) nanowires and find that disorder can even extend the topologically nontrivial phase in the relevant parameter space, creating topological order where none would be expected in a single channel system. Finally, in the Appendix, we give detailed derivations of our analytical results.

\section{TOPOLOGICAL ORDER IN DISORDERED QUASI-1D WIRES}

In this section, we investigate the topological properties of long quasi-1D RSW and PW TS nanowires (see Fig. 11. The quasiparticles in RSW nanowires are described by the following Bogoliubov-de Gennes (BdG) Hamiltonian:

$$
H=\left(\frac{\mathbf{p}^{2}}{2 m}+V(x)-\mu+\alpha(\mathbf{p} \times \sigma)\right) \tau_{z}+B \sigma_{x}+\Delta \tau_{x},
$$

where $\mathbf{p}=\left(p_{x}, p_{y}\right), V(x)$ is the on-site potential, $\mu$ is the chemical potential measured from the bottom of the band of the relevant lead, $\alpha$ is the spin-orbit coupling (SOC) strength, $B$ is the Zeeman field and $\Delta$ is the $s$ wave superconducting gap ${ }^{19}$. $\sigma_{i}\left(\tau_{i}\right)$ are the Pauli matrices acting on the spin (electron-hole) space. In the limit of large $B$, the different spins form completely separate bands and the low energy limit is an effective $p$-wave superconducting Hamiltonian of the form

$$
H=\left(\frac{\mathbf{p}^{2}}{2 m}+V(x, y)-\mu\right) \tau_{z}+\frac{1}{2} \sum_{i=x, y}\left\{\Delta_{i}, p_{i}\right\} \tau_{i} .
$$

We assume a spatially homogeneous effective SOC strength over the $\mathrm{PW}$ nanowire, i.e. $\Delta_{x}=\Delta_{y}=$ $\Delta_{\text {eff }}$, reducing the anticommutator term in Eq. (2) to $\frac{1}{2} \sum_{i=x, y}\left\{\Delta_{i}, p_{i}\right\} \tau_{i}=\Delta_{\text {eff }} \mathbf{p} \cdot \boldsymbol{\tau}$. This effective SOC strength is related to the corresponding RSW superconducting gap by $\Delta_{\text {eff }}=\Delta \alpha / \sqrt{B^{2}-\Delta^{218}}$. We consider both PW and RSW cases with disorder in the following subsections, assuming a Gaussian disorder of the form $\left\langle V(\mathbf{r}) V\left(\mathbf{r}^{\prime}\right)\right\rangle=\gamma \delta\left(\mathbf{r}-\mathbf{r}^{\prime}\right)$ for $\mathbf{r}, \mathbf{r}^{\prime}$ in the wire. Here, $\gamma$ is the disorder strength and $\langle V(\mathbf{r})\rangle=0$.

The Hamiltonians in Eqs. (1) and (2) are both in Altland-Zirnbauer (AZ) symmetry class D in two dimensions 59 with a $\mathbb{Z}_{2}$ topological number. In the absence of spin-orbit coupling (SOC) along the $y$-direction, the Hamiltonian in Eq. (2) also possesses a chiral symmetry, placing it into class $\mathrm{BDI}^{42150}$, having a $\mathbb{Z}$ topological number. (1D wires trivially satisfy this condition.) In the thin wire limit $(W \ll \xi), \Delta_{y} \ll \hbar / m W$ chiral symmetry breaking terms are $\mathcal{O}\left((m \alpha W / \hbar)^{2}\right)$ and can be neglected. The system has an approximate chiral symmetry $50+52$, placing the wire in the BDI class. We refer to a nanowire in this limit as a quasi-1D wire. The chiral topological number $Q_{\text {chiral }} \in \mathbb{Z}$ and the class-D topological number are related as $Q_{\mathbb{Z}_{2}}=-1^{Q_{\text {chiral }} 31}$. In order to understand this relation, consider a clean quasi-1D wire, where each conduction channel in the normal state can be treated as an effective 1D BDI superconducting wire, possibly sustaining Majorana end states. Then, $\left|Q_{\text {chiral }}\right|$ counts the number of channels that support Majorana states. For such a wire with a broken chiral symmetry, these Majorana states pairwise hybridize at each end to form ordinary fermions. Thus, if $Q_{\text {chiral }}$ is odd (i.e. $Q_{\mathbb{Z}_{2}}=-1$ ), an unpaired Majorana state remains and the wire is in a topologically nontrivial state. The relevant topological index that counts the number of Majorana end states are obtained through Lyapunov exponents $31 / 39 / 42 / 46$. In the disordered case, the comparison of a Lyapunov exponent with $\xi^{-1}$ determines whether that exponent contributes to the overall chiral topological index $Q_{\text {chiral }}$. We employ this method in the following sections to derive the topological phase diagram in our analytical calculations. We note in passing that while we work in $2 \mathrm{D}$ in this Manuscript, our methods and our general results should also apply to 3D cases with an appropriately calculated mean free path (MFP).

For our numerical simulations, we take the TB form of the Hamiltonians in Eq. (1) and (2) on a wire of length $L \gg l_{\mathrm{MFP}}, \xi$ where $l_{\mathrm{MFP}}$ is the MFP, having metallic leads $(\alpha=0, \Delta=0$ and $V(x, y)=0$ on the leads). We plot the phase diagram that we obtain from the numerics over the whole bandwidth, which is $8 t$ for the PW case with $t$ as the hopping parameter $t=\hbar^{2} / 2 m a^{2}$ and $a$ is the TB lattice spacing. (The bandwidth of the RSW case depends on the Zeeman field.) As the low energy limit of the TB model corresponds to the parabolic dispersion regime, the low $\mu$ regions in our plots yield the parabolic dispersion results. Fulga et al. derive the topological quantum number of disordered multichannel wires for various symmetry classes from the scattering matrices of the wires 31 . For a semi-infinite wire in the BDI symmetry class, the topological charge $Q$ is given by $Q=\operatorname{det}(r)$ where $r$ is the reflection matrix. (In this 


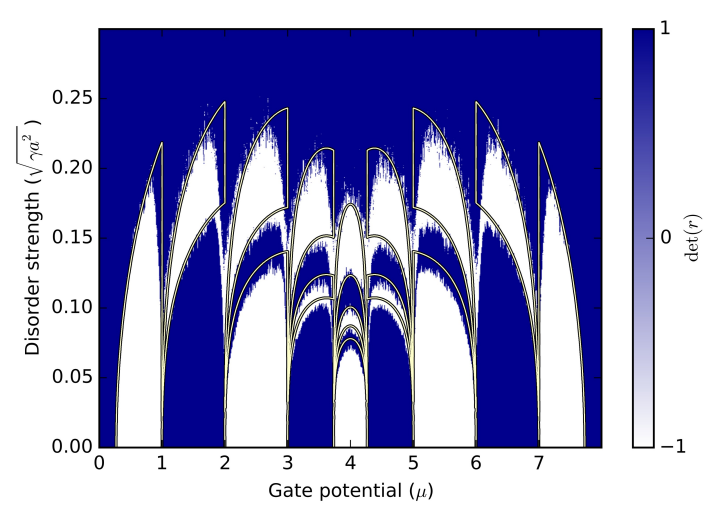

FIG. 2. (Color online) $\mu$ vs. $\sqrt{a^{2} \gamma}$ vs. $Q$ for a quasi-1D PW wire with dimensions $W=4 a$ and $L=60000 a$ ( $L$ used only in the numerical tight-binding code) and with $\alpha=0.01 \hbar / m a$, where $a$ is the tight-binding lattice spacing. The blue-white colors in the background are obtained numerically with a tight-binding method whereas the black-yellow solid lines are obtained using Eq. (3) with Eq. (6). The fuzziness at higher disorder values, which disappears as $L$ is increased, is due to finite size effects. No fitting parameters were used.

limit, the eigenvalues of the reflection matrix must be equal to \pm 1 to satisfy unitarity.) We use this relation to calculate $Q$ in our numerical TB simulations using the Kwant library 53 .

\section{A. Disordered quasi-1D $p$-wave wire}

We now investigate the effects of disorder on the topological order in a quasi-1D PW wire having the Hamiltonian in Eq. (2). The superconducting coherence length $\xi$ is given by $\xi=\hbar / m \Delta_{\text {eff. }}$ In order to solve the Schrödinger equation $H \Psi=E \Psi$ at $E=0$ we follow Ref. ${ }^{46}$ and off-diagonalize the Hamiltonian and apply an imaginary gauge transformation. This allows us to reexpress $Q_{\text {chiral }}$ in terms of normal state transport properties, called Lyapunov exponents $\Lambda_{n}$, which in essence specify the localization lenghts of eigenfunctions at a given energy in a multimode wire ${ }^{54}$. The result for the chiral topological index for a dirty wire is ${ }^{42}$ :

$$
Q_{\text {chiral }}=\sum_{n=1}^{N_{\text {open }}} \Theta\left(\xi-\frac{1}{\Lambda_{n}}\right),
$$

where $N_{\text {open }}=\left\lfloor W / \pi \sqrt{2 m \mu / \hbar^{2}}\right\rfloor$ is the number of open channels for the corresponding clean nanowire of width $W$ in the normal state at energy $\mu$ and $\lfloor x\rfloor$ yields the largest $n \leq x$ with $n \in \mathbb{Z}$. Note that the Lyapunov exponents are self averaging as $L \rightarrow \infty$, with a mean value given by

$$
\bar{\Lambda}_{n}=n /\left(N_{\mathrm{open}}+1\right) l_{\mathrm{MFP}}
$$

where $l_{\mathrm{MFP}}$ is the MFP of the disordered wire $\mathrm{s}^{54}$ and $n \in$ $1 \ldots N_{\text {open }}$.

In a clean wire, Majorana modes appear if $N_{\text {open }}$ is odd and Majorana states fuse to form ordinary Dirac fermions if $N_{\text {open }}$ is even. This behavior survives up to a finite disorder strength. Further increase of the disorder strength gives a series of transitions between non-trivial and trivial topological phases as $\Lambda_{n}$ increase and cross $\xi^{-1}$. Hence, the number of possible transitions for a disordered wire is equal to the number of open channels $N_{\text {open }}$ of the clean wire in its normal state for a given value of the chemical potential (see Fig. 2).

In order to obtain $l_{\mathrm{MFP}}^{-1}$, we employ Fermi's golden rule to obtain the inverse momentum lifetime $\left\langle\tau_{k \rightarrow k^{\prime}}^{-1}\right\rangle$ (see Appendix) first for a quadratic dispersion relation $\varepsilon(k)=\hbar^{2} k^{2} / 2 m$ to obtain the ensemble-averaged inverse momentum decay length $\left\langle l_{\operatorname{MFP}\left(k_{x}, n \rightarrow k_{x}^{\prime}, m\right)}^{-1}\right\rangle$. Summing over the possible transitions, we obtain

$$
l_{\mathrm{MFP}}^{-1}=\frac{4 m^{2} \gamma}{\hbar^{4} \pi k_{F}} \alpha_{N}^{-1}
$$

where $k_{F}=\sqrt{2 m \varepsilon / \hbar^{2}}$ is the Fermi wavevector and $\alpha_{N}^{-1}$ is a dimensionless number whose detailed form is given in Eq. A.5.

Note that this expression applies for quadratic dispersion only. Hence, the numerical TB calculations deviate from this result for regions away from the bottom of the band. In order to include the effects of band structure analytically and to be able to make a comparison of our formula with numerical results, we also analytically calculate the MFP for the tight-binding dispersion relation $\varepsilon\left(k_{x}\right)=2 t\left(2-\cos \left(k_{x} a\right)-\cos (n \pi a / W)\right)$ and obtain

$$
\left(l_{\mathrm{MFP}}^{\mathrm{TB}}\right)^{-1}=\frac{\gamma}{N_{\mathrm{open}} W a t^{2}}\left(\alpha_{N}^{\mathrm{TB}}\right)^{-1} .
$$

The corresponding number of channels is given by $N_{\text {open }}=\lfloor(W / \pi a) \arccos (1-\varepsilon / 2 t)\rfloor$ for $0<\varepsilon<4 t$ and by $N_{\text {open }}=\lfloor(W / \pi a) \arccos (1-(4-\varepsilon / 2 t))\rfloor$ for $4 t<\varepsilon<$ $8 t$. The details of the dimensionless number $\left(\alpha_{N}^{\mathrm{TB}}\right)^{-1}$ is given in Eq. A.8.

\section{B. Disordered quasi-1D $s$-wave wire}

Here, we focus on the experimentally relevant quasi1D RSW wires decribed by the Bogoliubov-de Gennes Hamiltonian in Eq. (1). As mentioned above, in the single channel limit, the Hamiltonian in Eq. (1) is in symmetry class $\mathrm{BDI}^{46}$ supporting a $\mathbb{Z}$ topological index $Q_{\text {chiral }}$. For a quasi $1 \mathrm{D}$ wire, the system has an approximate chiral symmetry $50 \mid 52$. Following the treatment detailed in the appendix of 4 , we extend the topological charge formula for the single channel to a quasi-1D case by summing the contribution of each Lyapunov exponent to the overall chiral topological charge. For the single channel case, we 


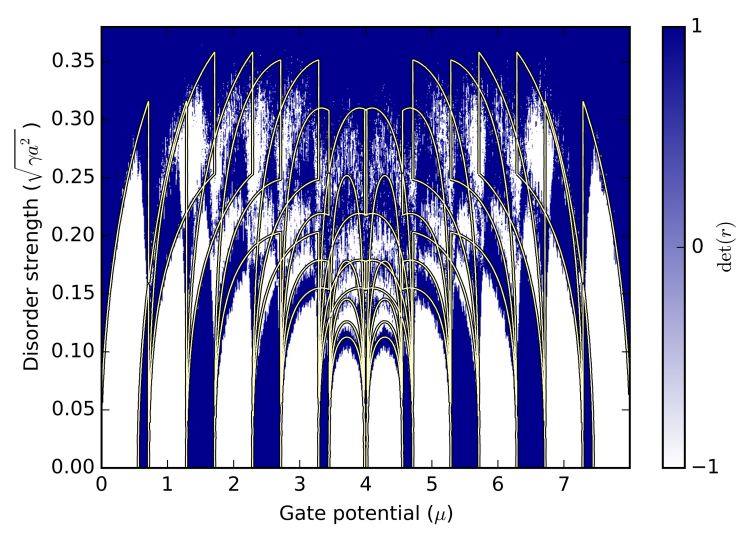

FIG. 3. (Color online) $\mu$ vs. $V_{0}=\sqrt{a^{2} \gamma}$ vs. $Q$ for a quasi1D RSW wire. The black-yellow lines, which represent topological boundaries, are obtained analytically using Eq. (9). The background blue-white colors are obtained using tightbinding numerical simulations with $L=60000 a$. In both cases, $W=4 a, \alpha=0.015 \hbar / m a, \Delta=0.20 t$ and $B=0.35 t$, where $t=\hbar^{2} / 2 m a^{2}$ is the tight-binding hopping parameter and $a$ is the TB lattice spacing. The fuzziness of the numerical results at higher disorder values, which disappears as $L$ is increased, is again due to finite size effects.

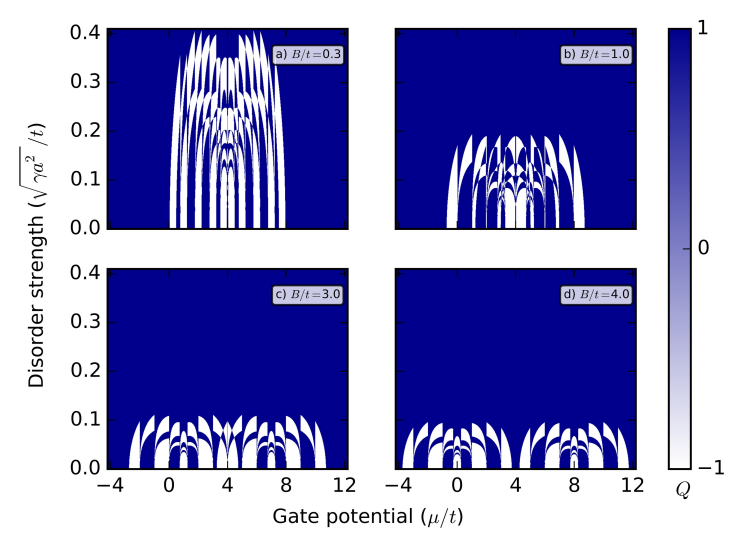

FIG. 4. (Color online) $\mu$ vs. $V_{0}=\sqrt{a^{2} \gamma}$ vs. $Q_{\mathbb{Z}_{2}}$ for a quasi-1D RSW wire for different $B$, obtained analytically using Eq. (8). a), b) Low magnetic field $B \gtrsim \Delta$ limit requires a full RSW model and topological order can survive up to high disorder strengths. c), d) The spin-polarized system can be described by a PW model and topological order is completely destroyed with less disorder. Here, $W=4 a, \alpha=0.015 \hbar / m a$ and $\Delta=0.20 \hbar^{2} / 2 m a^{2}$, and $a$ is the tight-binding lattice spacing.

reproduce the result of Ref $\frac{46}{}$ :

$$
\begin{aligned}
Q_{1 \mathrm{D}}= & \operatorname{sgn}\left(\xi_{\mathrm{eff}}-\frac{1}{|\Lambda(\mu+\epsilon)|}\right) \times \\
& \operatorname{sgn}\left(\xi_{\mathrm{eff}}-\frac{1}{|\Lambda(\mu-\epsilon)|}\right),
\end{aligned}
$$

where $\epsilon=\sqrt{B^{2}-\Delta^{2}}$ and the effective coherence length is $\xi_{\text {eff }}=\frac{\hbar \epsilon}{m \alpha \Delta}$. For a general multichannel wire we obtain:

$$
\begin{aligned}
Q_{\text {chiral }}= & \sum_{n=1}^{N_{\text {open }}} \Theta\left(\xi_{\text {eff }}-\frac{1}{\left|\Lambda_{n}(\mu+\epsilon)\right|}\right)- \\
& \sum_{n=1}^{N_{\text {open }}^{\prime}} \Theta\left(\xi_{\text {eff }}-\frac{1}{\left|\Lambda_{n}(\mu-\epsilon)\right|}\right)
\end{aligned}
$$

This formula suggests that the multichannel $s$-wave topological superconductor wire can be treated as two parallel PW multichannel topological superconductor wires with effective "spin polarized" chemical potentials $\mu_{\text {eff }}=\mu \pm \epsilon$. These two "wires" each have a corresponding number of Lyapunov exponents $N_{\text {open }}$ and $N_{\text {open }}^{\prime}$ (which need not be equal to each other) and a set of Lyapunov exponents $\Lambda_{n} \rightarrow \Lambda_{n}\left(\mu_{\text {eff }}\right)$. We caution the reader however that these "effective" wires have nothing to do with the channels of the clean wire as these modes are completely mixed due to disorder. We calculate $\Lambda_{n}$ and $Q_{\text {chiral }}$ as above with the appropriate substitutions and obtain the class D topological index as $Q=(-1)^{Q_{\text {chiral }}}$, which yields

$$
Q=\prod_{n, \pm} \operatorname{sgn}\left(\Lambda_{n}(\mu \pm \epsilon) \xi_{\mathrm{eff}}-1\right) .
$$

In Fig. 3, we compare our analytical result with the numerical TB simulations. We note the good agreement between Eq. 9 and the TB simulations all over the phase diagram. In Fig. 4 we see that increasing the magnetic field spin-polarizes the RSW wire as expected, ultimately separating the phase diagram into that of two PW wires in the high magnetic field limit (Fig. 4 c) and 4d)). More interesting physics occurs in the intermediate regime where both spin components play a role, depicted in Fig. 3, 4 a) and $4 \mathrm{~b}$ ). In this regime, it is no longer possible to reduce the system to a simple spinless PW model, but it's still possible to get non-trivial topological phases that support Majorana states. As the disorder strength increases, series of topological transitions occur, similar to the PW wire. However, unlike the PW wire, the number of transitions is given by $N_{\text {open }}+N_{\text {open }}^{\prime}-1$ rather than the $N_{\text {open }}$ transitions for PW wire. The increasing fragility of the wire under rising $B$ and further spin polarization is evident in Fig. 4, as the maximum disorder value above which no topological transition is possible decreases from Fig. 4 a) to Fig. 4 d).

For completeness, we also investigate how the topological phase diagram as a function of magnetic field strength $B$ and chemical potential $\mu$ changes as disorder is increased. In Fig. 5 [Fig. 6], the analytically calculated phase diagram of a wire with $W=a[W=4 a]$ is plotted with increasing disorder. We note that the phase diagram gets fragmented as number of channels are increased. Moreover, the structure of the phase diagram becomes increasingly complicated as the number of patches of topological regions increase. Perhaps more importantly, we also note that for a given amount of disorder, there is a 


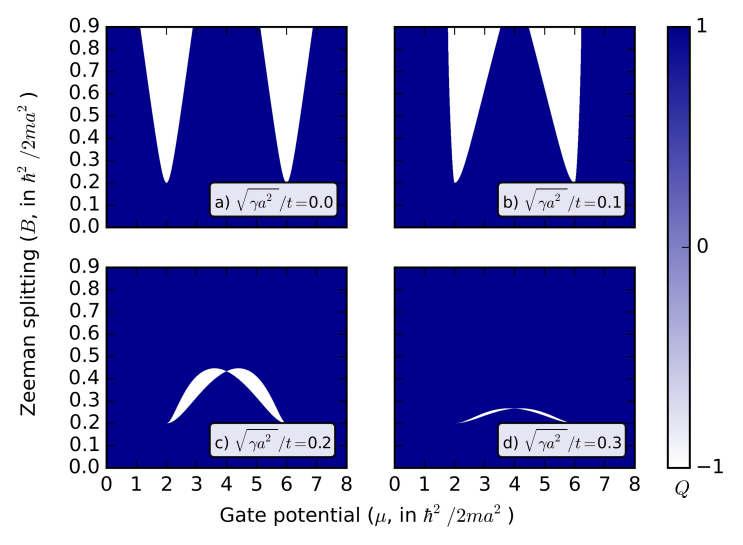

FIG. 5. (Color online) $\mu$ vs. $B$ vs. $Q_{\mathbb{Z}_{2}}$ for varying disorder strengths for an RSW TS with Gaussian disorder, analytically calculated using Eq. 8 for a single-channel system. The parameters used are $\alpha=0.015 \hbar / m a$ and $\Delta=0.2 \hbar^{2} / 2 m a^{2}$, where $a$ is the lattice spacing.

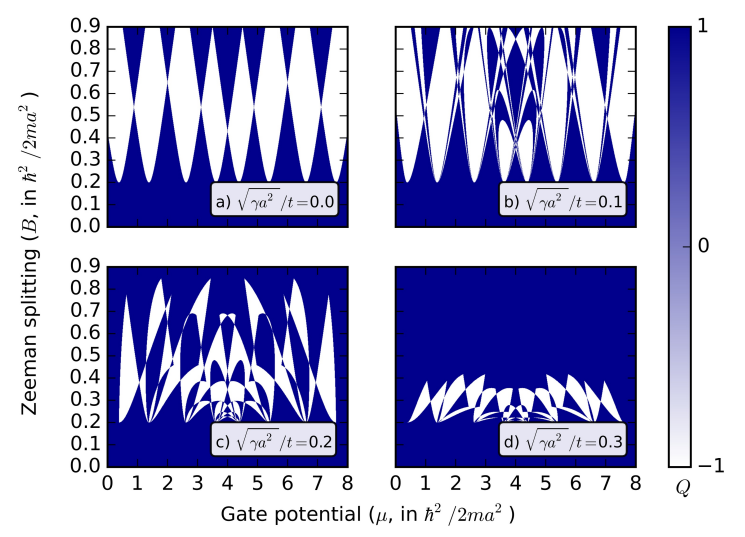

FIG. 6. (Color online) $\mu$ vs. $B$ vs. $Q_{\mathbb{Z}_{2}}$ for varying disorder strengths for an RSW TS with Gaussian disorder, analytically calculated using Eq. (8) for a four-channel TB system. Subfigure c) matches the numerical data shown in Fig. 7. The parameters used are $\alpha=0.015 \hbar / m a$ and $\Delta=0.2 \hbar^{2} / 2 m a^{2}$, where $a$ is the lattice spacing.

maximum Zeeman field $B_{\max }$ above which no topological order is present. The reason is that in our numerical simulations, the localization length is not a monotonous function of energy: it grows (with increasing energy) until the middle of the band, and after that it decreases as the energy comes closer to the band edge. This places an upper magnetic field limit to topological regions since the superconducting coherence length monotonically increases with $B$. However for a quadratic dispersion, the upper limit is infinite. We stress that the upper limit discussed here has a different origin than that discussed by Ref.55 for finite-length wires. Our numerical results for the parameters of Fig. 6 is presented in Fig. 7. The patchy structure is quite visible and well-correlates with

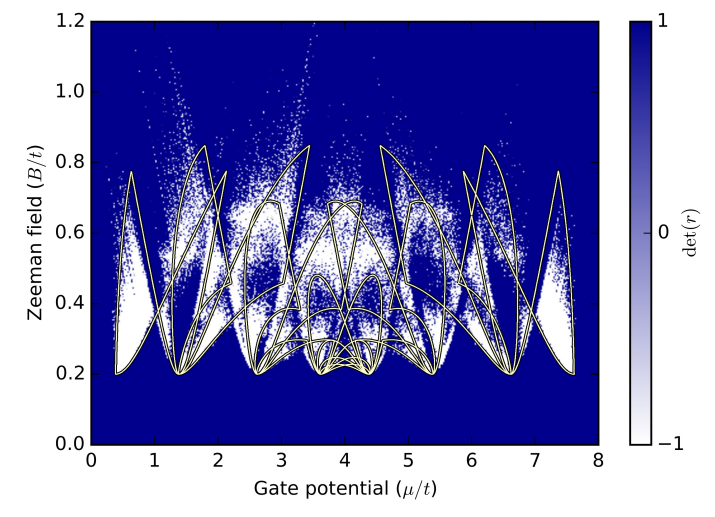

FIG. 7. (Color online) $\mu$ vs. $B$ vs. $Q_{\mathbb{Z}_{2}}$ for a four-channel system (compare with Fig. 6.) The background blue-white colors are obtained using a numerical tight-binding simulation with $L=20000 a$ and $W=4 a$, while the black-yellow lines are obtained analytically using Eq. 88. Here, $V_{0}=\sqrt{\gamma a^{2}}=0.2 t$, $\alpha=0.015 \hbar / m a$ and $\Delta=0.2 t$, where $t=\hbar^{2} / 2 m a^{2}$ is the tight-binding hopping parameter and $a$ is the tight-binding lattice spacing.

the analytical prediction.

\section{CONCLUSION}

In summary, we investigated the effect of disorder in multichannel $p$-wave and $s$-wave topological superconductor nanowires. Our analysis showed that disorder in a multichanneled wire creates reentrant topological states in both the $p$-wave and the $s$-wave limit. We derived analytical formulas for topological phase diagrams and compared our results to numerical tight-binding simulations. We showed that the opening and closing of transport gaps (as opposed to electronic band gaps) due to disorder can also drive a system to a topologically nontrivial state. Our formulas also apply to lower magnetic field regions in the phase diagram, where the $p$-wave approximation breaks down. As experimental wires always have some disorder and can easily gated to have many conduction channels, we expect our results to be experimentally accessible.

\section{ACKNOWLEDGMENTS}

This work was supported by funds of the Erdal İnönü chair, by TÜBİTAK under grant No. 110T841, by the Foundation for Fundamental Research on Matter (FOM) and by Microsoft Corporation Station Q. IA is a member of the Science Academy-Bilim Akademisi-Turkey; BP, AT and ÖB thank The Science Academy-Bilim Akademisi-Turkey for the use of their facilities throughout this work. 


\section{Appendix: Mean free path}

We consider a long wire along the $x$-axis, having a length of $L$ and a width of $W$ along the $y$-direction and metallic leads at the end, with a Gaussian disorder of the form $\left\langle V(\mathbf{r}) V\left(\mathbf{r}^{\prime}\right)\right\rangle=\gamma \delta\left(\mathbf{r}-\mathbf{r}^{\prime}\right)$. We obtain the ensemble average of the matrix element between the $n^{\text {th }}$ and $m^{\text {th }}$ transverse channels as $\boldsymbol{k}\left(k_{x}, n\right) \rightarrow \boldsymbol{k}^{\prime}\left(k_{x}^{\prime}, m\right)$ as

$$
\left\langle\left|V_{k k^{\prime}}\right|^{2}\right\rangle=\frac{\gamma}{L W}\left(1+\frac{\delta_{n, m}}{2}\right)
$$

We then use Fermi's golden rule to calculate the inverse lifetime of a momentum state $k, \tau_{k \rightarrow k^{\prime}}^{-1}$ :

$$
\begin{aligned}
\left\langle l_{\operatorname{MFP}\left(k_{x}, n \rightarrow k_{x}^{\prime}, m\right)}^{-1}\right\rangle= & \left(\frac{1}{\hbar} \frac{\partial \varepsilon_{k}}{\partial k_{x}}\right)^{-1} \times \frac{2 \pi}{\hbar} \frac{\gamma}{L W} \times \\
& \left(1+\frac{\delta_{n, m}}{2}\right) \rho\left(\varepsilon_{k^{\prime}}\right) .
\end{aligned}
$$

where $\varepsilon_{k}$ gives the dispersion relation and $\rho\left(\varepsilon_{k}\right)$ is the density of states. We then sum over the initial and final states $k^{\prime}$ in Eq. A.2 to obtain the total inverse MFP:

$$
\left\langle l_{\mathrm{MFP}}^{-1}\right\rangle=\sum_{k_{x}, k_{y} ; k_{x}^{\prime}, k_{y}^{\prime}}\left\langle l_{\mathrm{MFP}\left(k_{x}, n \rightarrow k_{x}^{\prime}, l\right)}^{-1}\right\rangle
$$

We first apply Eq. A.3 to a free electron dispersion of the form $\varepsilon(k)=\hbar^{2} k^{2} / 2 m=\hbar^{2} / 2 m\left(k_{n, x}^{2}+n^{2} \pi^{2} / W^{2}\right)$ for $n \in 1, \ldots, N_{\text {open }}$ where $N_{\text {open }}$ is the number of open channels in a clean wire with width $W$ at energy $\varepsilon$. The resulting total ensemble-averaged inverse MFP is

$$
\begin{aligned}
\left\langle l_{\mathrm{MFP}}^{-1}\right\rangle= & \sum_{n=1}^{N_{\text {open }}} \sum_{l=1}^{N_{\text {open }}} \int \frac{d k_{n, x}^{\prime}}{\pi / L} \frac{m^{2}}{\hbar^{4}} \frac{2 \gamma W}{L \pi}\left(1+\frac{\delta_{n l}}{2}\right) \frac{\pi}{W} \times \\
& \frac{\delta\left(k_{l, x}^{\prime} \pm \sqrt{2 m \varepsilon / \hbar^{2}-l^{2} \pi^{2} / W^{2}}\right)}{\sqrt{2 m \varepsilon / \hbar^{2}-n^{2} \pi^{2} / W^{2}} \sqrt{2 m \varepsilon / \hbar^{2}-l^{2} \pi^{2} / W^{2}}} \\
= & \frac{4 m^{2} \gamma}{\hbar^{4} \pi k_{F}} \alpha_{N}^{-1},
\end{aligned}
$$

where $k_{F}=\sqrt{2 m \varepsilon / \hbar^{2}}$ is the Fermi wavevector,

$$
\begin{aligned}
\alpha_{N}^{-1}= & \frac{3 N_{\text {open }}}{2} \sum_{n=1}^{N_{\text {open }}}\left(\frac{W^{2} k_{F}^{2}}{\pi^{2}}-n^{2}\right)^{-1}+ \\
& 2 N_{\text {open }} \sum_{n=1}^{N_{\text {open }}} \sum_{l>n}^{N_{\text {open }}} f_{n} f_{l},
\end{aligned}
$$

and $f_{n}=\left(\frac{W^{2} k_{F}^{2}}{\pi^{2}}-n^{2}\right)^{-\frac{1}{2}}$, in agreement with Eq.(8) in the supporting online material of Ref ${ }^{42}$.

We now derive the MFP for a TB dispersion relation given by

$$
\varepsilon\left(k_{x, n}\right)=2 t\left(2-\cos \left(k_{x, n} a\right)-\cos (n \pi a / W)\right) .
$$

The number of channels is given by $N_{\text {open }}=$ $\lfloor(W / \pi a) \arccos (1-\varepsilon / 2 t)\rfloor$ for $0<\varepsilon<4 t$ and by $N_{\text {open }}=\lfloor(W / \pi a) \arccos (1-(4-\varepsilon / 2 t))\rfloor$ for $4 t<\varepsilon<$ $8 t$. The resulting disorder-averaged inverse MFP reads:

$$
\left\langle\left(l_{\mathrm{MFP}}^{\mathrm{TB}}\right)^{-1}\right\rangle=\frac{\gamma}{N_{\text {open }} W a t^{2}}\left(\alpha_{N}^{\mathrm{TB}}\right)^{-1}
$$

where the dimensionless $\left(\alpha_{N}^{\mathrm{TB}}\right)^{-1}$ is given by

$$
\begin{aligned}
\left(\alpha_{N}^{\mathrm{TB}}\right)^{-1}= & \frac{3 N_{\text {open }}}{2} \sum_{n=1}^{N_{\text {open }}} \frac{1}{\sin ^{2}\left(k_{x, n} a\right)}+ \\
& 2 N_{\text {open }} \sum_{n=1}^{N_{\text {open }}} \sum_{l>n}^{N_{\text {open }}} f_{n}^{\mathrm{TB}} f_{l}^{\mathrm{TB}}
\end{aligned}
$$

where $f_{n}^{\mathrm{TB}}=\left(\sin ^{2}\left(k_{x, n} a\right)\right)^{-\frac{1}{2}}$ and $\sin \left(k_{x, n}\right)$ is obtained using Eq. A.6
* barisp@sabanciuniv.edu

† adagideli@sabanciuniv.edu

1 M. Z. Hasan and C. L. Kane, Rev. Mod. Phys. 82, 3045 (2010)

2 X. L. Qi and S. C. Zhang, Rev. Mod. Phys. 83, 1057 (2011)

3 M. Franz and L. Molenkamp, eds., Topological Insulators, 1st ed., Contemporary Concepts of Condensed Matter Science, Vol. 6 (Elsevier, 2013)

4 J. Alicea, Rep. Prog. Phys. 75, 076501 (2012)
5 M. Leijnse and K. Flensberg, Semicond. Sci. Technol. 27, 124003 (2012)

${ }^{6}$ C. W. J. Beenakker, Annu. Rev. Condens. Matter Phys. 4, 113 (2013)

7 A. Bernevig and T. Hughes, Topological Insulators and Topological Superconductors, 1st ed. (Princeton University Press, 2013)

8 S. Elliott and M. Franz, Rev. Mod. Phys. 87, 137 (2015)

9 A. Y. Kitaev, Ann. Phys. 303, 2 (2003) 
10 C. Nayak, S. H. Simon, A. Stern, M. Freedman, and S. Das Sarma, Rev. Mod. Phys. 80, 1083 (2008)

11 R. Jackiw and P. Rossi, Nucl. Phys. B 190, 681 (1981)

12 M. M. Salomaa, Phys. Rev. B 37, 9298 (1988)

13 G. Moore and N. Read, Nucl. Phys. B 360, 36296 (1991)

14 N. Read and D. Green, Phys. Rev. B 61, 10267 (2000)

15 D. A. Ivanov, Phys. Rev. Lett. 86, 268 (2001)

16 A. Y. Kitaev, Phys.-Usp. 44, 131 (2001)

17 J. Alicea, Phys. Rev. B 81, 125318 (2010)

18 R. M. Lutchyn, J. D. Sau, and S. Das Sarma, Phys. Rev. Lett. 105, 077001 (2010)

19 Y. Oreg, G. Refael, and F. von Oppen, Phys. Rev. Lett. 105, 177002 (2010)

20 Other proposals include $\sqrt{56} 68$

${ }^{21}$ V. Mourik, K. Zuo, S. M. Frolov, S. R. Plissard, E. P. A. M. Bakkers, and L. P. Kouwenhoven, Science 336, 1003 (2012)

22 A. Das, Y. Ronen, Y. Most, Y. Oreg, M. Heiblum, and H. Shtrikman, Nat. Phys. 8, 887 (2012)

23 M. T. Deng, C. L. Yu, G. Y. Huang, M. Larsson, P. Caroff, and H. Q. Xu, Nano Lett. 12, 6414 (2012)

${ }^{24}$ A. D. K. Finck, D. J. Van Harlingen, P. K. Mohseni, K. Jung, and X. Li, Phys. Rev. Lett. 110, 126406 (2013)

25 H. O. H. Churchill, V. Fatemi, K. Grove-Rasmussen, M. T. Deng, P. Caroff, H. Q. Xu, and C. M. Marcus, Phys. Rev. B 87, 241401 (2013)

26 S. Nadj-Perge, I. K. Drozdov, J. Li, H. Chen, S. Jeon, J. Seo, A. H. MacDonald, B. A. Bernevig, and A. Yazdani, Science 346, 602 (2014)

27 Other sources of ZBPs include Kondo effect, weak antilocalization and disorder-induced level crossings 25/40/69]72].

28 O. Motrunich, K. Damle, and D. Huse, Phys. Rev. B 63, 224204 (2001)

${ }^{29}$ I. A. Gruzberg, N. Read, and S. Vishveshwara, Phys. Rev. B 71, 245124 (2005)

30 A. R. Akhmerov, J. P. Dahlhaus, F. Hassler, M. Wimmer, and C. W. J. Beenakker, Phys. Rev. Lett. 106, 057001 (2011)

31 I. C. Fulga, F. Hassler, A. R. Akhmerov, and C. W. J. Beenakker, Phys. Rev. B 83, 155429 (2011)

32 A. C. Potter and P. A. Lee, Phys. Rev. B 83, 184520 (2011)

33 A. C. Potter and P. A. Lee, Phys. Rev. B 84, 059906 (2011)

34 T. D. Stanescu, R. M. Lutchyn, and S. Das Sarma, Phys. Rev. B 84, 144522 (2011)

35 P. W. Brouwer, M. Duckheim, A. Romito, and F. von Oppen, Phys. Rev. B 84, 144526 (2011)

36 J. D. Sau, S. Tewari, and S. Das Sarma, Phys. Rev. B 85, $064512(2013)$

37 A. M. Lobos, R. M. Lutchyn, and S. Das Sarma, Phys. Rev. Lett. 109, 146403 (2012)

${ }^{38}$ F. Pientka, A. Romito, M. Duckheim, Y. Oreg, and F. von Oppen, New J. Phys. 15, 025001 (2013)

39 W. DeGottardi, D. Sen, and S. Vishveshwara, Phys. Rev. Lett. 110, 146404 (2013)

40 P. Neven, D. Bagrets, and A. Altland, New J. Phys. 15, 055019 (2013)

41 J. D. Sau and S. Das Sarma, Phys. Rev. B 88, 064506 (2013)

42 M. T. Rieder, P. W. Brouwer, and İ. Adagideli, Phys. Rev. B 88, 060509 (2013)

${ }^{43}$ D. Chevallier, P. Simon, and C. Bena, Phys. Rev. B 88,
$165401(2013)$

44 W. DeGottardi, M. Thakurathi, S. Vishveshwara, and D. Sen, Phys. Rev. B 88, 165111 (2013)

45 P. Jacquod and M. Büttiker, Phys. Rev. B 88, 241409 (2013)

46 I. Adagideli, M. Wimmer, and A. Teker, Phys. Rev. B 89, 144506 (2014)

${ }^{47}$ H. Y. Hui, J. D. Sau, and S. Das Sarma, Phys. Rev. B 90, $064516(2014)$

48 F. Pientka, G. Kells, A. Romito, P. W. Brouwer, and F. von Oppen, Phys. Rev. Lett. 109, 227006 (2012)

49 A. Altland and M. R. Zirnbauer, Phys. Rev. B 55, 1142 (1997)

50 S. Tewari and J. D. Sau, Phys. Rev. Lett. 109, 150408 (2012)

51 M. T. Rieder, G. Kells, M. Duckheim, D. Meidan, and P. W. Brouwer, Phys. Rev. B 86, 125423 (2012)

52 M. Diez, J. P. Dahlhaus, M. Wimmer, and C. W. J. Beenakker, Phys. Rev. B 86, 094501 (2012)

53 C. W. Groth, M. Wimmer, A. R. Akhmerov, and X. Waintal, New J. Phys. 16, 063065 (2014)

54 C. W. J. Beenakker, Rev. Mod. Phys. 69, 731 (1997)

55 D. Rainis, L. Trifunovic, J. Klinovaja, and D. Loss, Phys. Rev. B 87, 024515 (2013)

56 T.-P. Choy, J. M. Edge, A. R. Akhmerov, and C. W. J. Beenakker, Phys. Rev. B 84, 195442 (2011)

57 M. Kjaergaard, K. Wölms, and K. Flensberg, Phys. Rev. B 85, 020503 (2012)

58 I. Martin and A. F. Morpurgo, Phys. Rev. B 85, 144505 (2012)

59 S. Nadj-Perge, I. K. Drozdov, B. A. Bernevig, and A. Yazdani, Phys. Rev. B 88, 020407 (2013)

60 B. Braunecker and P. Simon, Phys. Rev. Lett. 111, 147202 (2013)

61 F. Pientka, L. I. Glazman, and F. von Oppen, Phys. Rev. B 88, 155420 (2013)

62 J. Klinovaja, P. Stano, A. Yazdani, and D. Loss, Phys. Rev. Lett. 111, 186805 (2013)

63 S. Nakosai, Y. Tanaka, and N. Nagaosa, Phys. Rev. B 88, 180503 (2013)

64 M. M. Vazifeh and M. Franz, Phys. Rev. Lett. 111, 206802 (2013)

65 Y. Kim, M. Cheng, B. Bauer, R. M. Lutchyn, and S. Das Sarma, Phys. Rev. B 90, 060401 (2014)

66 J. Rönynen and T. Ojanen, arXiv:cond-mat.str$\mathrm{el} / 1412.5814$ (2014)

67 J. Lee, T. Neupert, Z. J. Wang, A. H. MacDonald, A. Yazdani, and B. A. Bernevig, arXiv:cond-mat.meshall/1501.00999 (2015)

68 B. Scharf and I. Žutić, Phys. Rev. B 91, 144505 (2015)

69 E. J. H. Lee, Z. Jiang, R. Aguado, G. Katsaros, C. M. Lieber, and S. De Franceschi, Phys. Rev. Lett. 109, 186802 (2012)

70 J. Liu, A. C. Potter, K. T. Law, and P. A. Lee, Phys. Rev. Lett. 109, 267002 (2012)

71 D. Bagrets and A. Altland, Phys. Rev. Lett. 109, 227005 (2012)

72 D. I. Pikulin, J. P. Dahlhaus, M. Wimmer, H. Schomerus, and C. W. J. Beenakker, New J. Phys. 14, 125011 (2012) 